DESY $80 / 131$

December 1980

COMPUTER SIMULATION OF THE BEAM-BEAM INTERACTION

by

A. Piwinski 
DESY $80 / 131$

December 1980

\section{Computer Simulation of the Beam-Beam Interaction}

by

A. Piwinski

\section{ABSTRACT}

The beam-beam interaction is simulated on a digital computer taking into account all three oscillation modes with quantum fluctuation and damping. It is shown that small asymmetries in phase advance between the interaction points due to machine imperfections and spurious dispersions at the interaction points are the main causes of an increase of the beam height. The simulations agree with measurements at the storage ring PETRA, and first experiments gave an improvement of the luminosity as predicted by the simulations. 


\section{Introduction}

Since an analytical description of the blow-up due to the beambeam interaction does not yet exist several approaches have been made to simulate this effect on a digital computer by following the two- or three-dimensional motion of protrons ${ }^{l}$ and electrons with quantum fluctuation and damping $2,3,4$ ) over a large number of revolutions. This report especially includes the effect of certain machine distortions.

The exact space charge forces of an opposing bunch were calculated for a two-dimensional grid of points and then interpolated quadratically for each passage of a particle. The ratio of the beam height to the beam width of the opposing bunch was kept constant in these calculations, i.e. we consider here the "weak - strong" case.

Between the interaction points the betatron oscillations and the synchrotron oscillation were transformed linearly. Damping and quantum fluctuation were taken into account in all three dimensions.

The simulations show that the blow-up is essentially caused by disturbances of the ideal machine. Different betatron phase advances between the interaction points and spurious horizontal dispersions at the interaction points have a drastic effect on the blow-up. Horizontal and vertical asymmetries of the betatron phases between the interaction points excite additional resonances which become important with increasing number of interaction points. Also a small horizontal dispersion at the interaction points enlarges the blow-up whereas a small vertical dispersion shows no strong influence.

The longitudinal motion of the interaction point which is seen by an individual particle due to its synchrotron oscillation is approximately taken into account. The change of the amplitude function is negligible for present PETRA-parameters. The change of the betatron phases between two interaction points due to the longitudinal motion, however, has an influence on the blow-up and is always included. 


\section{Space Charge Forces}

The transverse field of a bunch with a gaussion particle distribution is easily obtained if one assumes relativistic particle energies. The potential is then given by electrostatic potential theory and has the well known form

$\Phi(x, z)=\frac{Q}{4 \pi \varepsilon_{0}} \int_{0}^{\infty} \frac{\exp \left(-\frac{x^{2}}{2 \sigma_{x}^{2}+q}-\frac{z^{2}}{2 \sigma_{z}^{2}+q}\right)}{\sqrt{2 \sigma_{x}^{2}+q} \sqrt{2 \sigma_{z}^{2}+q}} d q$

with $Q=$ line charge density, $\varepsilon_{0}=$ dielectric constant

$\sigma_{x, z} \quad=$ horizontal and vertical standard deviation

For a numerical solution of the integral it is convenient to replace the variable q by

$$
s=2 \frac{\sigma_{x}^{2}-\sigma_{z}^{2}}{2 \sigma_{x}^{2}+q} \quad\left(\sigma_{x}>\sigma_{z}\right)
$$

The integration interval then becomes finite and the change of the betatron angles due to the passage through an opposing bunch is given by

$$
\begin{aligned}
& \Delta x^{\prime}=-\frac{2 \pi \xi_{x} x}{\beta_{x o}(l-v)} \int_{0}^{1-v^{2}} \exp \left\{-a s-\frac{b s}{I-s}\right\} \frac{d s}{\sqrt{1-s}} \\
& \Delta z^{\prime}=-\frac{2 \pi \xi_{z} v z}{\beta_{z o}(l-v)} \int_{0}^{1-v^{2}} \exp \left\{-a s-\frac{b s}{l-s}\right\} \frac{d s}{(1-s)^{3 / 2}}
\end{aligned}
$$


with

$$
\begin{gathered}
v=\frac{\sigma_{z}}{\sigma_{x}} \\
a=\frac{1}{2} \frac{x^{2}}{\sigma_{x}^{2}-\sigma_{z}^{2}} \\
b=\frac{1}{2} \frac{z^{2}}{\sigma_{x}^{2}-\sigma_{z}^{2}}
\end{gathered}
$$

$\xi_{x, z}=$ horizontal and vertical space charge parameter

$\beta_{x o, z o}=$ horizontal and vertical amplitude function

The integrals were calculated for $75 \times 200$ points with a distance of $0.2 \sigma_{x}$ and $0.2 \sigma_{z}$ between the points

\section{Damping and Quantum Fluctuation}

The damping of the 3 oscillations is included in the linear transformation of the 6 particle coordinates between the interaction points. The damping reduces the amplitudes according to the radiation damping, and the partition numbers of the horizontal and vertical betatron damping and the synchrotron damping are 1,1 and 2 , respectively.

The quantum fluctuation is simulated by changing the coordinates by random kicks at each interaction point. The random kicks have a constant distribution and their mean value is zero. Since in a real machine the kicks occur at arbitrary betatron phase distances from the interaction point, in the simulation $x$ and $x$ ' and also $z$ and $z^{\prime}$ are changed at the same time but by different random kicks. This means that there is no coupling and the vertical beam height is assumed to be produced by a spurious vertical dispersion in the magnets. A linear coupling of horizontal and vertical betatron oscillations due to rotated quadrupoles was also tentatively included but showed no effect on the blow-up. 
4. Simulations

The following PETRA parameters are used:

$\sigma_{x 0} / \sigma_{z 0}=\beta_{x 0} / \beta_{z 0}=15, \sigma_{S} / \beta_{z 0}=0.1, Q_{x}=25.2, Q_{S}=0.07$

with $\sigma_{S}=$ longitudinal standard deviation

$Q_{x, s}=$ horizontal betatron and synchrotron wave number

The number of particles times the number of turns investigated are given in the following table:

\begin{tabular}{l|c|c|c} 
E/GeV & 7 & 11.3 & 17.9 \\
\hline 2 IP & - & $125 * 36000$ & $125 * 11000$ \\
4 IP & $64 * 100000$ & $64 * 33000$ & $125 * 9000$ \\
8 IP & - & $36 * 30000$ & $125 * 9000$ \\
$\begin{array}{l}\text { revolutions } \\
\text { per betatron } \\
\text { damping time }\end{array}$ & 12500 & 3000 & 750 \\
\end{tabular}

At the start the particles are distributed equidistant in phase and gaussian in amplitude. The obtained blow-up is characterized by

$$
\frac{1}{\sigma_{z 0}} \sqrt{\left\langle z^{2}\right\rangle}=\frac{1}{\sigma_{z 0}} \sqrt{\frac{1}{N} \sum_{i=l}^{N} z_{i}^{2}}
$$

$N$ includes all particles at all interaction points and all revolutions after 4 damping times.

The same quantity is calculated for the horizontal coordinates, but the increase of the beam width is much smaller than the increase of the beam height and is, therefore, not considered here. In investigating the influence of machine disturbances on the blow-up asymmetries of the betatron phase advances between the interaction points and spurious dispersions at the interaction points were introduced as follows: 
The maximum asymmetry in phase advance per section was $\delta Q= \pm 0.075$ for both, horizontal and vertical betatron oscillations. Such an asymmetry can be produced by orbit bumps which contain sextupoles. Asymmetries of $\delta Q \approx \pm 0.02$ occur easily if the orbit is not completely corrected.

The maximum dispersion was $11 \mathrm{~cm}$ for a horizontal amplitude function of $2.25 \mathrm{~m}$ at the interaction point. This value corresponds to spurious dispersions measured in the straight sections after an orbit correction. The width produced by synchrotron oscillations is then $20 \%$ of the width produced by betatron oscillations, but the total width is only $2 \%$ larger than the betatron width.

Figs. I to 13 show the increase in beam height as a function of the vertical betatron wave number $Q_{z}$, $i . e$. the vertical betatron frequency in units of the revolution frequency. The influence of various parameters as the space charge parameter $\xi_{x, z}$ $\left(\approx \Delta Q_{x, z}\right)$, the horizontal and vertical phase asymmetry, the horizontal dispersion, the number of interaction points and the energy, i.e. the damping time, can be seen.

\section{Conclusion}

With these distortions the simulations show the same blow-up as observed in PETRA. Thus, the simulations can help to find a better working point and to avoid dangerous distortions. At $Q_{z}=23.1$, for instance, the blow-up should become appreciably smaller if it is possible to reduce the asymmetries and to compensate the horizontal dispersion at the interaction points. First experiments with PETRA at $14 \mathrm{GeV}$ have shown that, for this working point, after carefully correcting the orbit and minimizing the horizontal dispersion the blow up could be reduced by about a factor of 2 . Thus the luminosity could be increased by about a factor of 2 at $14 \mathrm{GeV}$. A further enlargement of the luminosity seems possible if the control of the asymmetries and dispersions can be improved. 


\section{Acknowledgement}

The author is grateful to Mr. J. Kewisch for his help in preparing the computer programs.

\section{References}

1. E.D. Courant, in "Nonlinear Dynamics and the Beam-Beam Interaction", AIP Conference Proceedings No. 57, P. 194-201 (1979).

2. J. Tennyson, University of California, Berkeley (1980) (unpublished).

3. S. Peggs and R. Talman, llth International Conf. High Energy Accelerators, CERN 1980, P. 754 (1980).

4. A. Piwinski, llth International Conf. High Energy Accelerators, CERN 1980, P.751 (1980). 


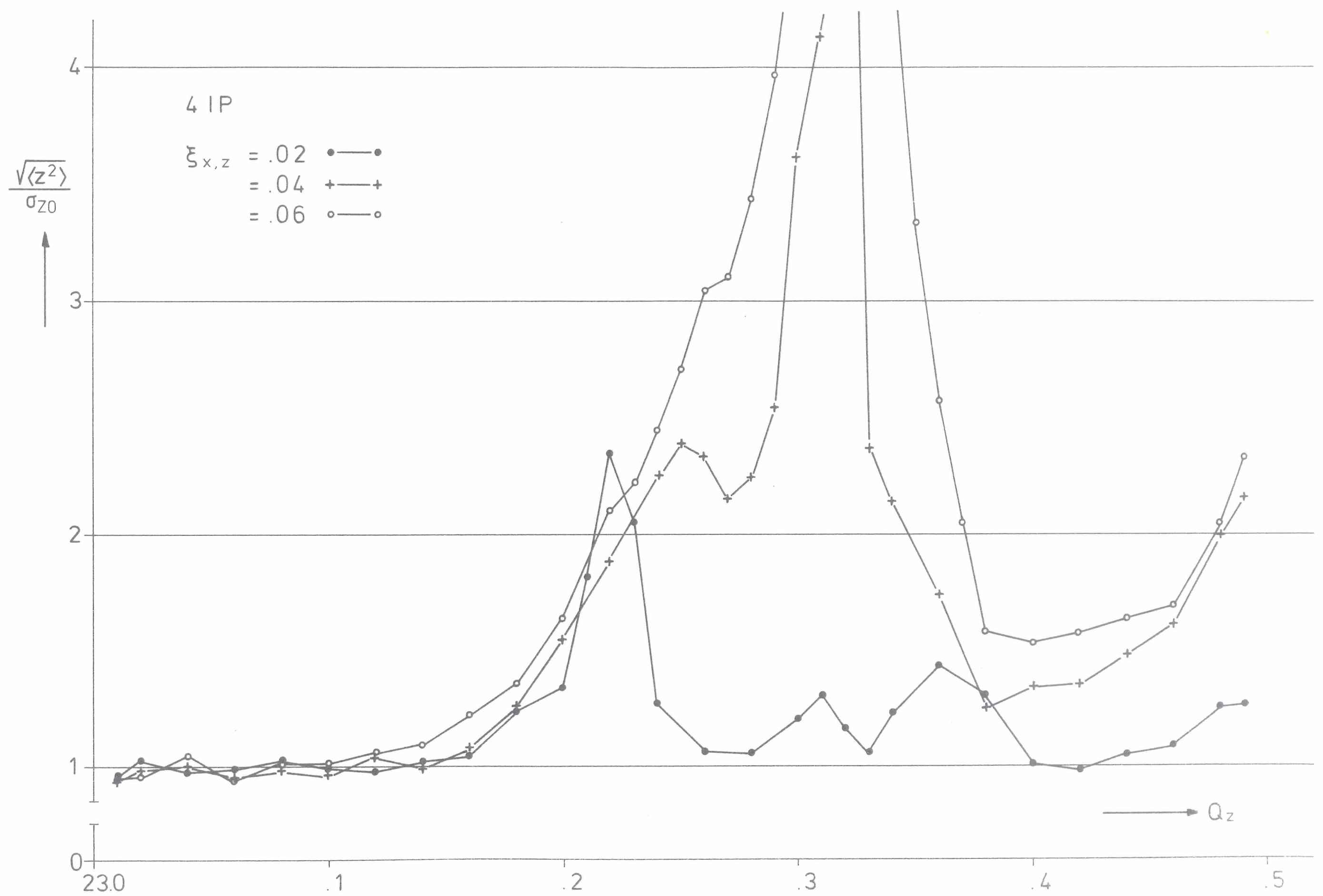

Fig. I Increase of beam height without distortions (E=1l.3 GeV) 


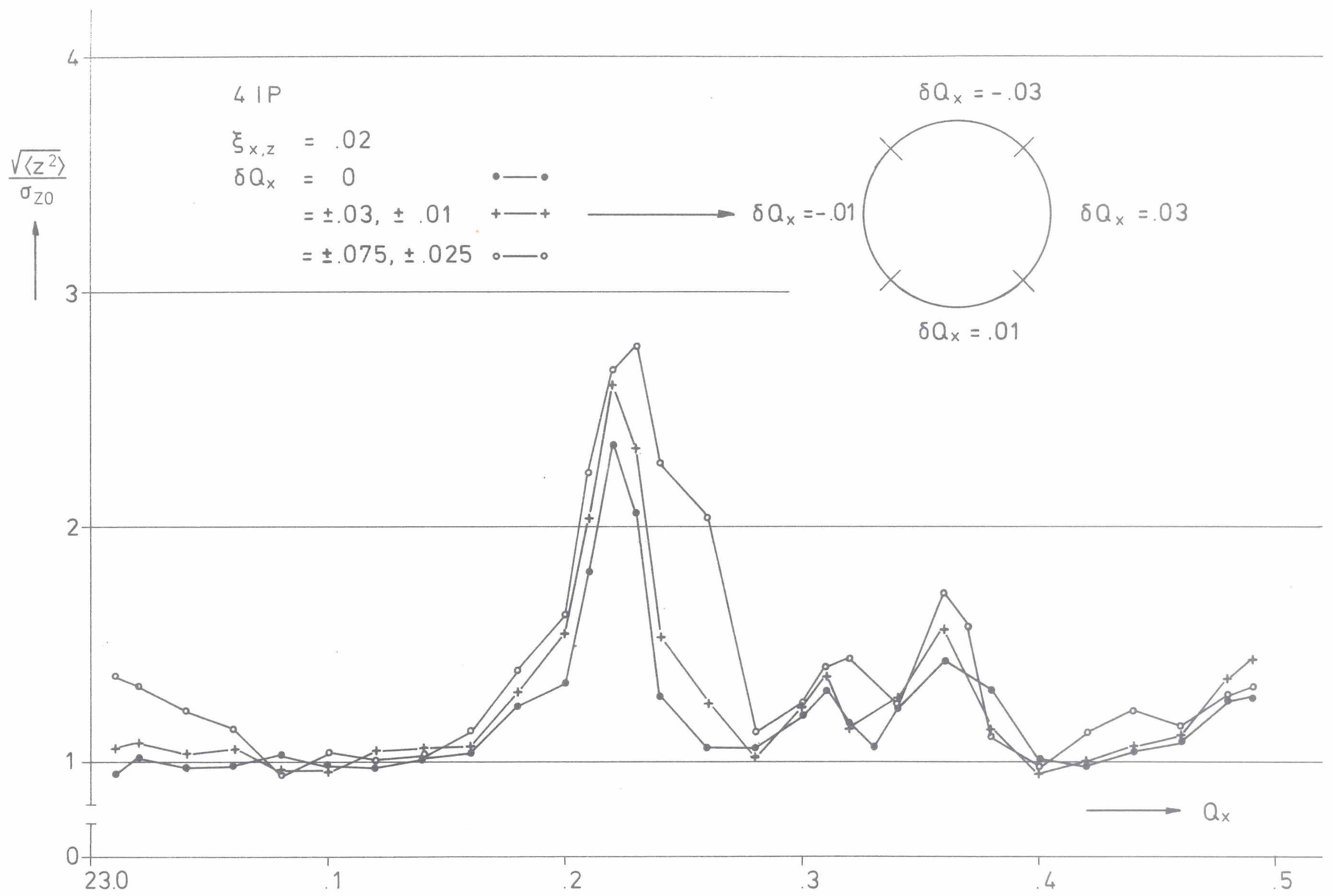

Fig. 2 Increase of beam height with horizontal asymmetries (E=ll.3 GeV) 


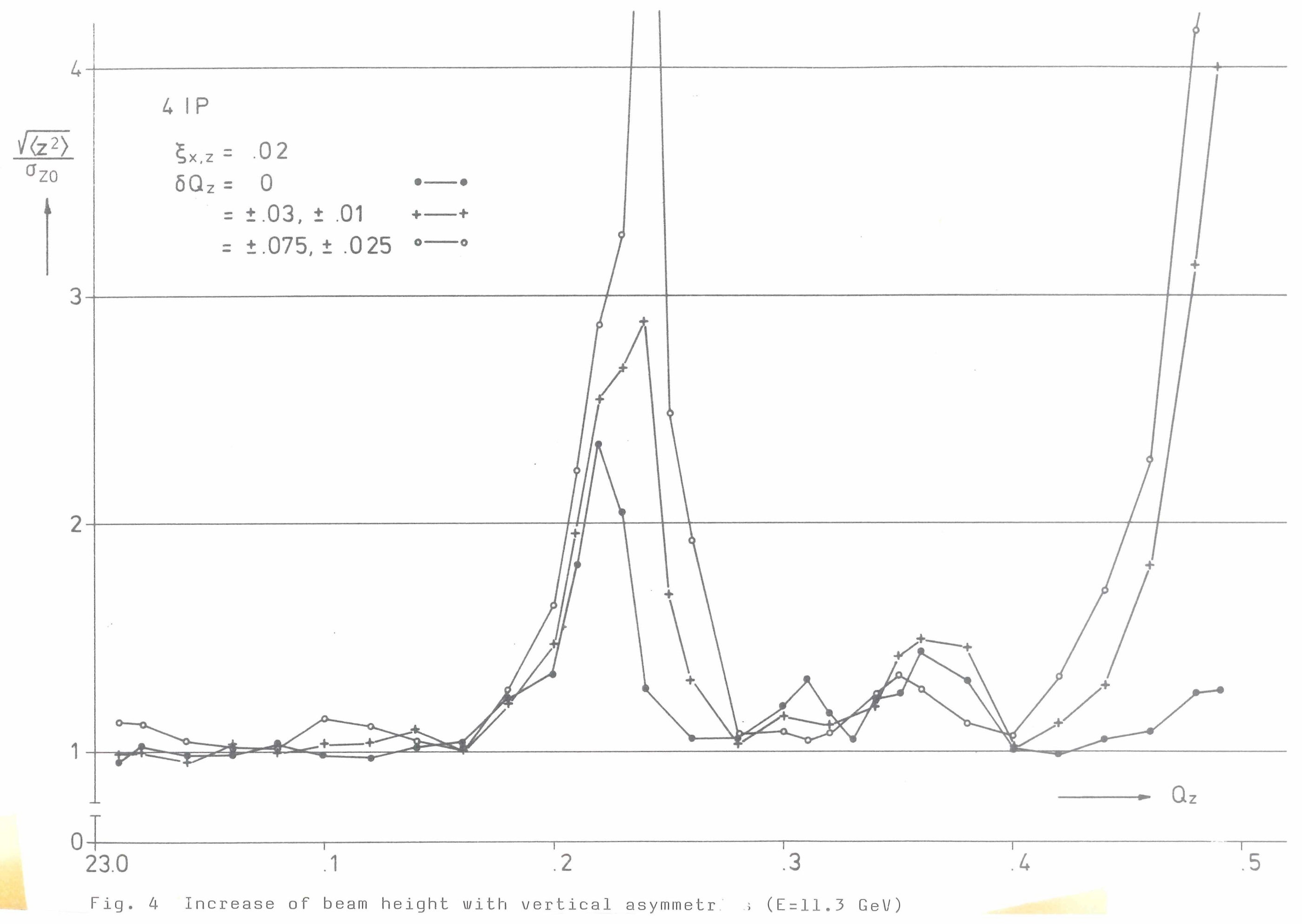




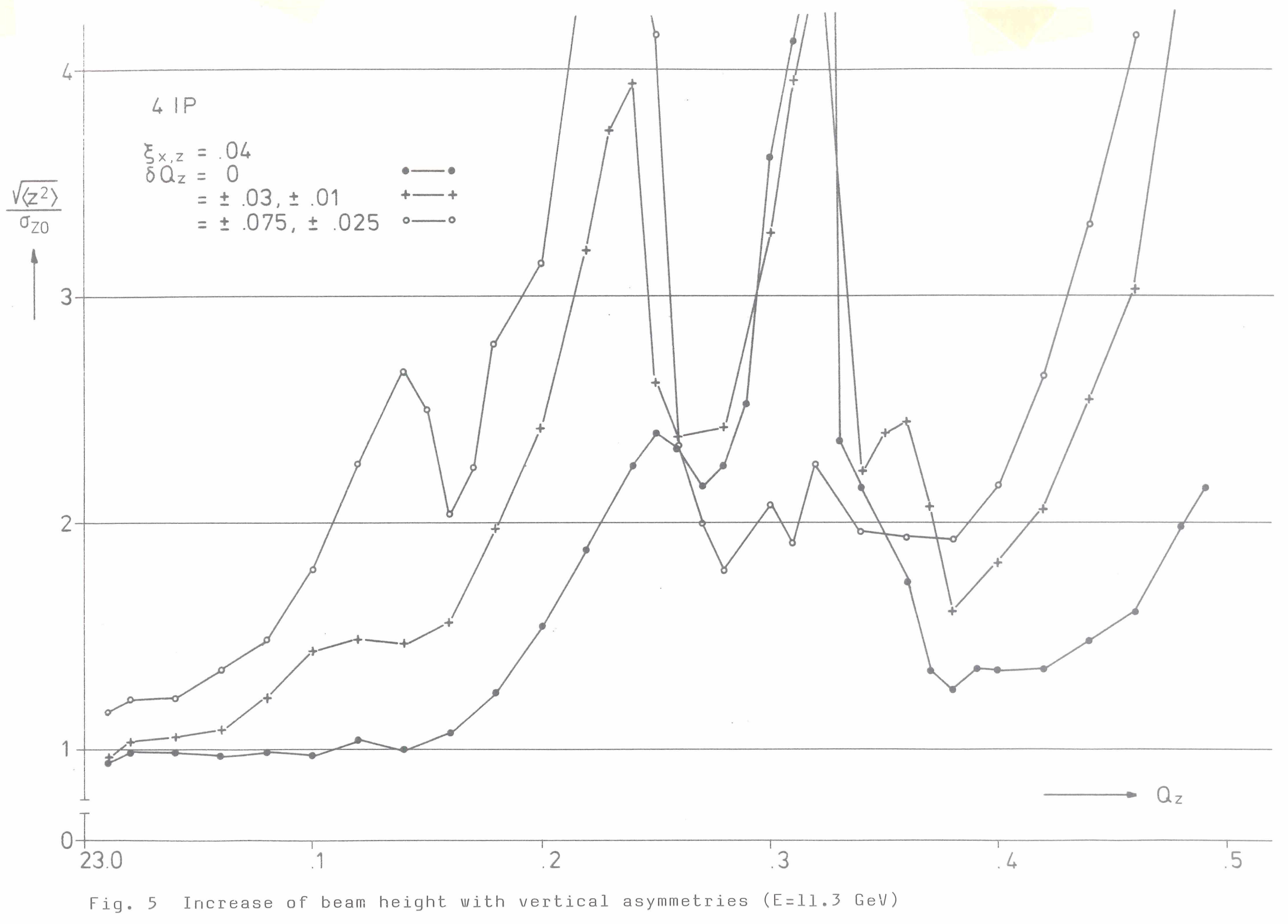




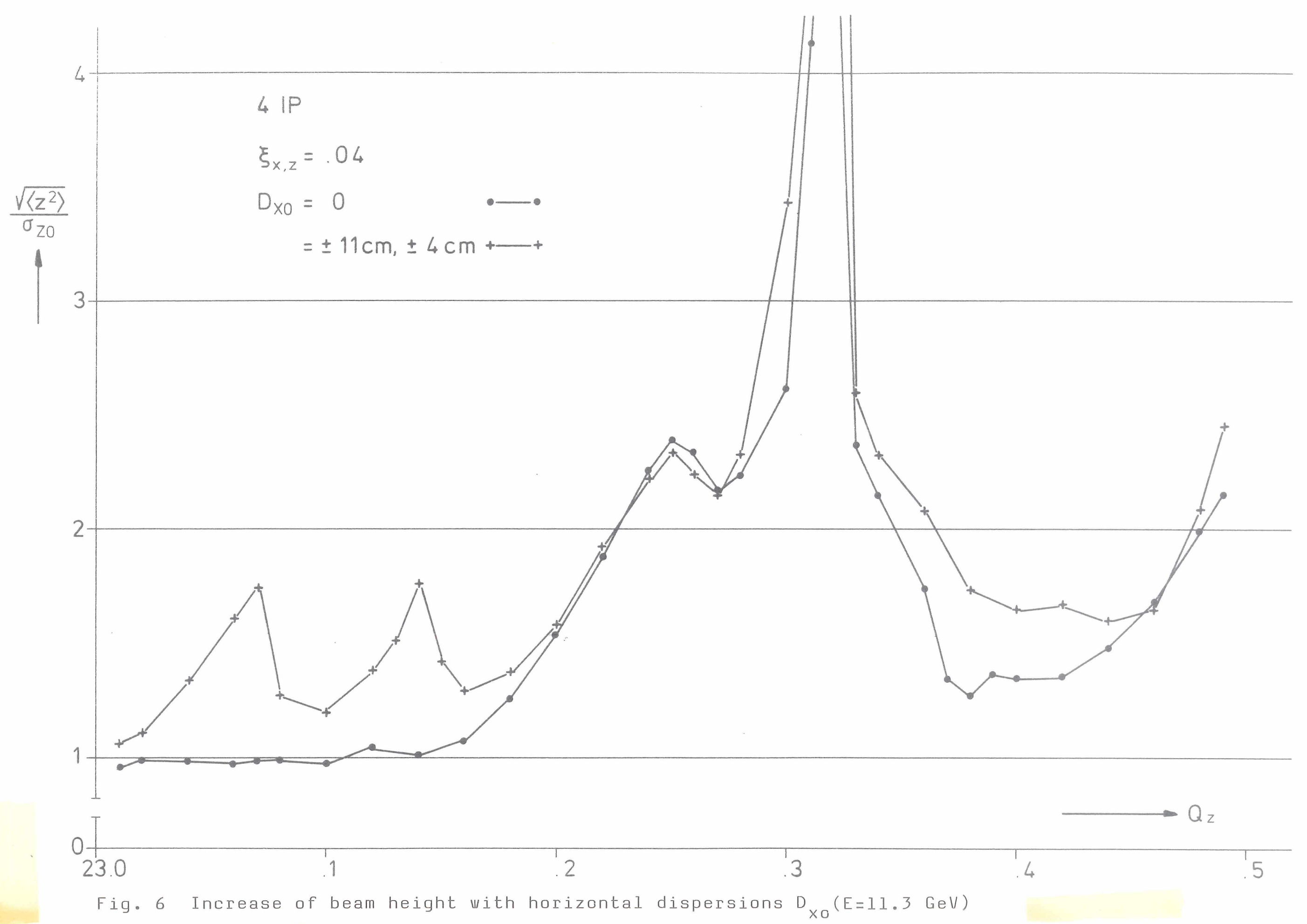




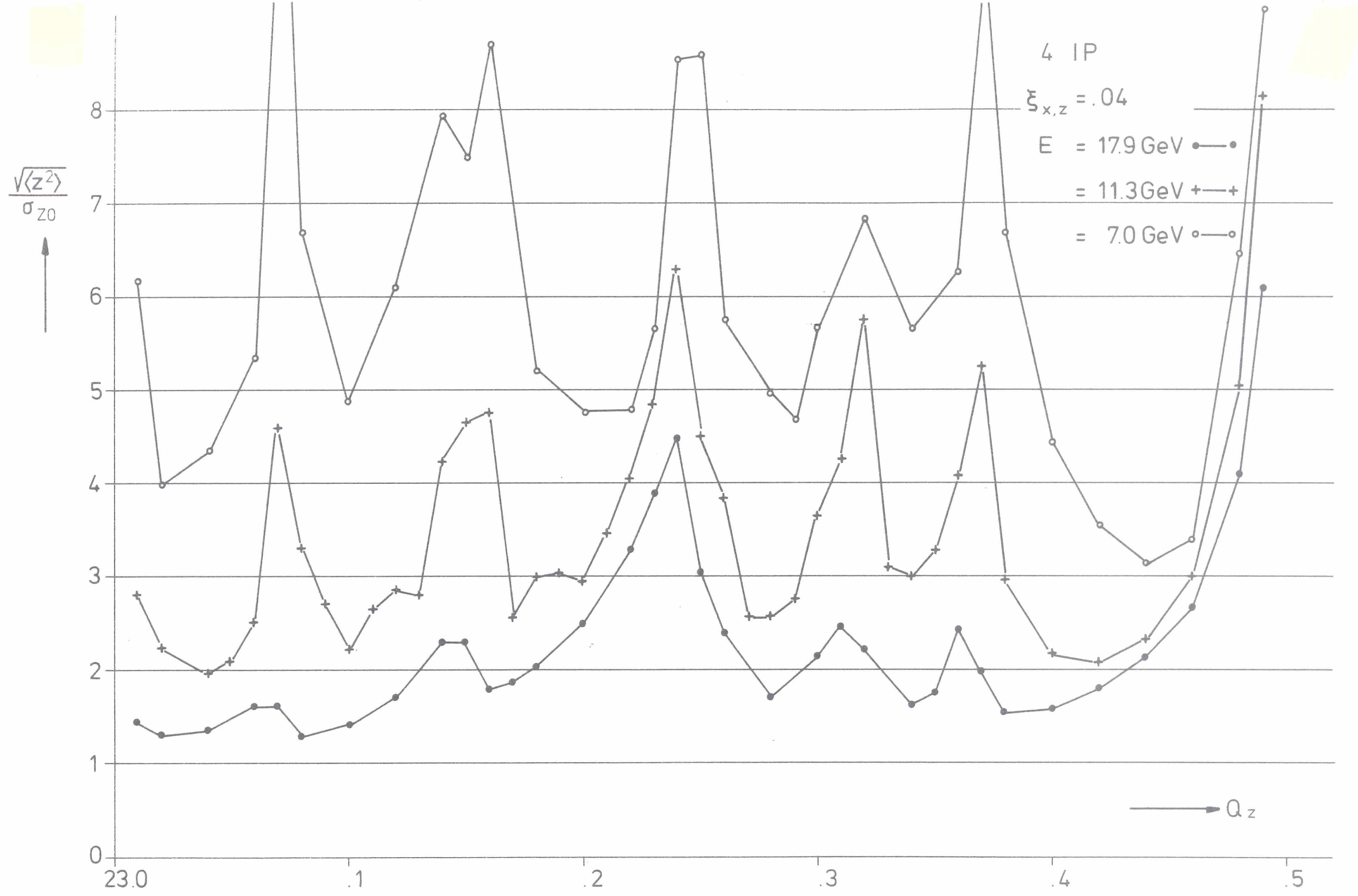

Fig. 9 Increase of beam height with distortions $\left(\delta Q_{x, z}= \pm .03, \pm .01, D_{x 0}= \pm I l c m, \pm 4 c m\right)$ for different energjes 


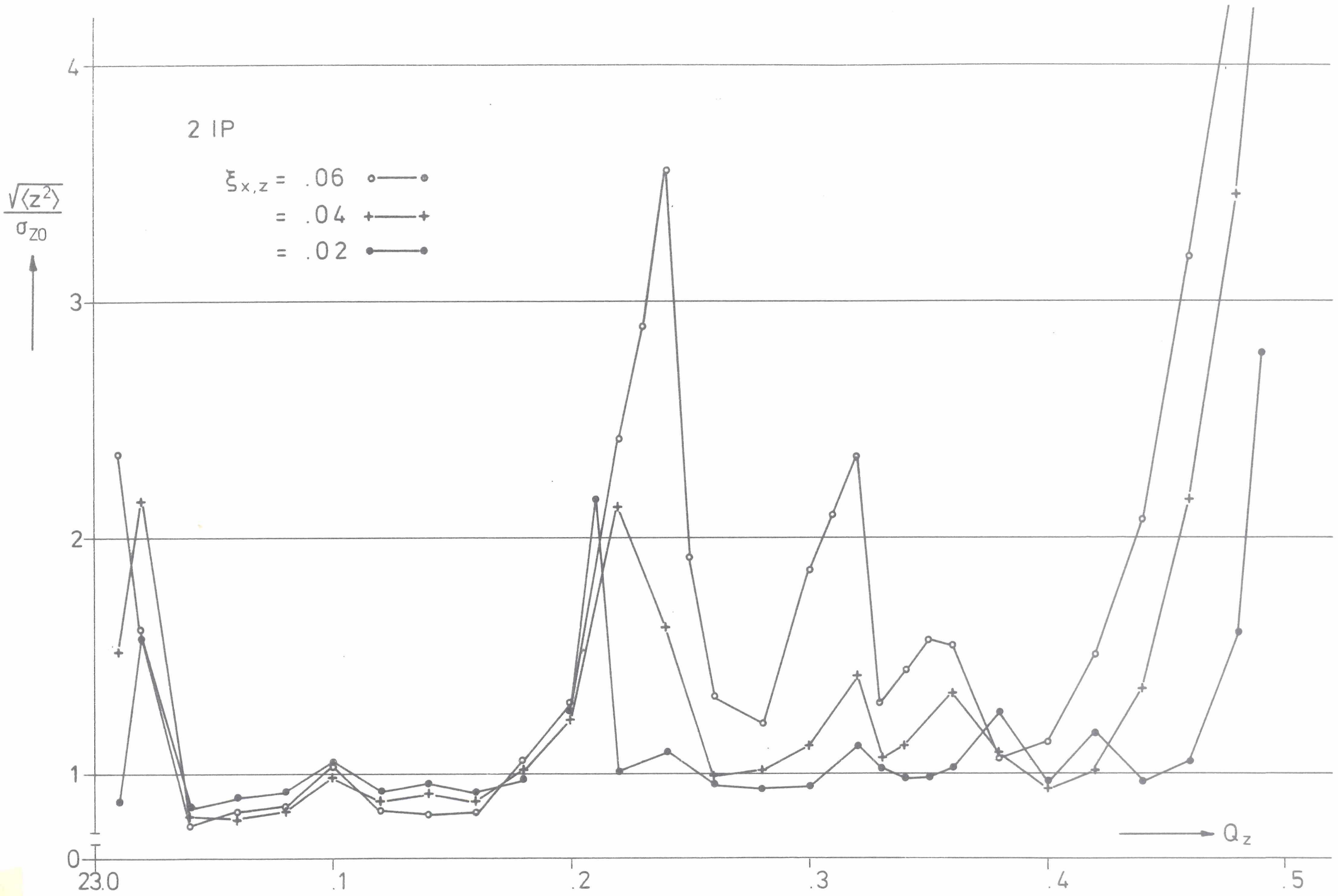

Fig. 10 Increase of beam height without distortions ( $E=1 l .3$ GeV) 


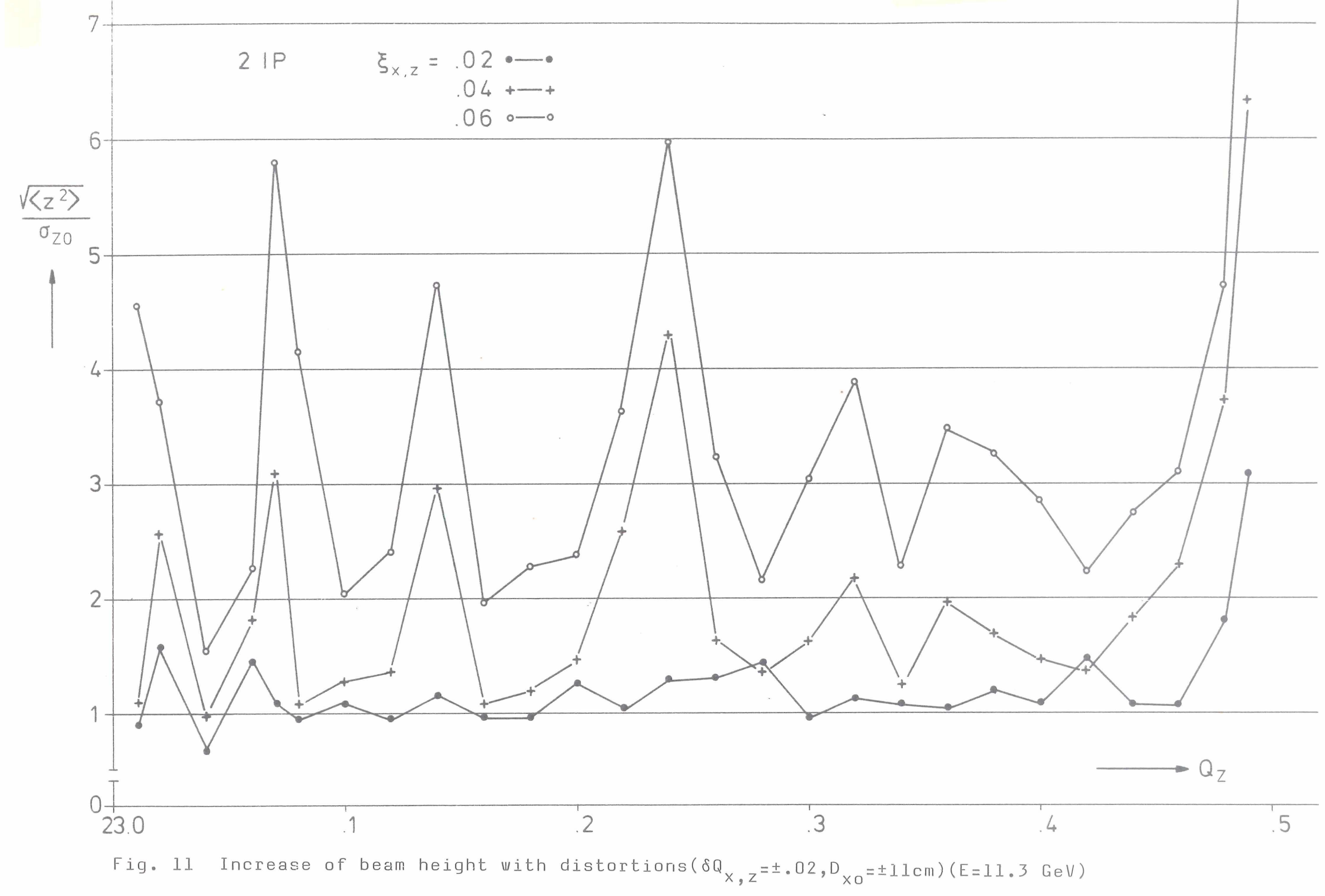




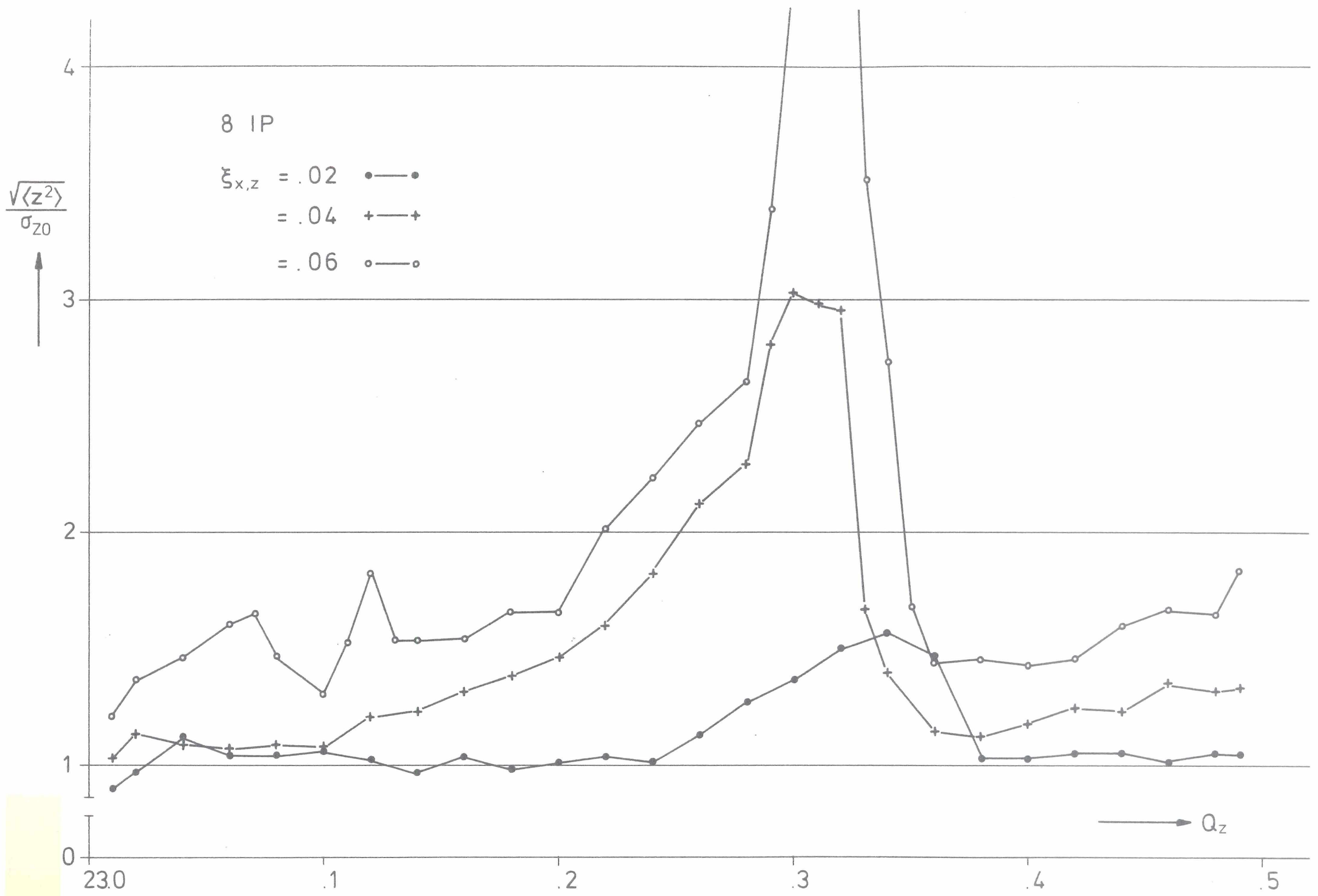

Fig. 12 Increase of beam height without distortions (E=ll.3 GeV) 
